\title{
Swainsonine inhibits growth and potentiates the cytotoxic effect of paclitaxel in hepatocellular carcinoma in vitro and in vivo
}

\author{
NAN YOU ${ }^{1 *}$, WEIHUI LIU ${ }^{1,2^{*}}$, TAO WANG $^{1 *}, \mathrm{RU} \mathrm{JI}^{1^{*}}, \mathrm{XING}_{\text {WANG }}{ }^{1}$, \\ ZHENBING GONG ${ }^{1}$, KEFENG DOU $^{1}$ and KAISHAN TAO ${ }^{1}$ \\ ${ }^{1}$ Department of Hepatobiliary Surgery, Xijing Hospital, Fourth Military Medical University, Xi'an 710032; \\ ${ }^{2}$ PLA Center of General Surgery, General Hospital of Chengdu Army Region, Chengdu 610083, P.R. China
}

Received June 13, 2012; Accepted August 23, 2012

DOI: $10.3892 /$ or.2012.2035

\begin{abstract}
Swainsonine, an extract from Astragalus membranaceus, exhibits broad inhibition of growth and pro-apoptotic activity in a number of tumor types. However, the underlying mechanism involved remains unclear. To investigate the effects and mechanisms of swainsonine on hepatocellular carcinoma (HCC), we performed experiments on HepG2, SMCC7721, Huh7 and MHCC97-H human hepatoma and HL-7702 human hepatocyte cells. We observed that swainsonine significantly inhibited the viability of human hepatoma cells in a dose- and time-dependent manner, but did not affect human hepatocytes. Due to their highly proliferative and tumorigenic nature, we selected MHCC97-H cells as a model system to examine. Swainsonine significantly inhibited MHCC 97-H cell growth by causing cell cycle arrest at the G0/G1 phase and the induction of apoptosis. Blockage of G0/G1 phase was accompanied by a decrease in cyclins (D1 and E) and cyclin-dependent kinases (Cdk2 and Cdk4) and an increase in the Cdk inhibitors p21 and p27. Furthermore, swainsonine enhanced the apoptosis of MHCC97-H cells with the induction of the upregulation of Bax and the downregulation of $\mathrm{Bcl}-2$, whereas the expressionof Fas and Fas- $\mathrm{L}$ remained almost unchanged. These changes were
\end{abstract}

Correspondence to: Dr Kaishan Tao and Dr Kefeng Dou, Department of Hepatobiliary Surgery, Xijing Hospital, Fourth Military Medical University, Xi'an 710032, P.R. China

E-mail: kaishantao@yeah.net

E-mail: kefengdou@yeah.net

*Contributed equally

Abbreviations: BSA, bovine serum albumin; Cdk, cyclindependent kinases; DMEM, Dulbecco's modified Eagle's medium; DMSO, dimethyl sulfoxide; FBS, fetal bovine serum; GAPDH, glyceraldehyde-3-phosphate dehydrogenase; HCC, hepatocellular carcinoma; MTT, 3-(4,5-dimethylthiazol-2-yl)-2,5-diphenyltetrazolium bromide; NF- $\kappa \mathrm{B}$, nuclear factor $\kappa \mathrm{B}$; PBS, phosphate-buffered saline; $\mathrm{PI}$, propidium iodide; TEM, transmission electron microscope

Key words: hepatocellular carcinoma, swainsonine, growth, paclitaxel accompanied by the enhanced cytoplasmic accumulation of nuclear factor $\kappa \mathrm{B}(\mathrm{NF}-\mathrm{\kappa B})$ with a concomitant decrease in the nuclear fraction. Importantly, swainsonine also potentiated the cytotoxic effects of paclitaxel in vitro and in vivo, in part, by restricting the paclitaxel-induced nuclear accumulation of $\mathrm{NF}-\kappa \mathrm{B}$. Taken together, these results suggest that swainsonine may be an important agent against HCC via directly inhibiting HCC cell growth and enhancing the responsiveness of HCC cells to paclitaxel.

\section{Introduction}

Hepatocellular carcinoma (HCC) is one of the most frequent causes of death from solid tumors in the world, particularly in China $(1,2)$. Currently, surgical intervention, including hepatic resection and liver transplantation, is the only therapy considered to provide a cure. However, only a few patients are diagnosed early enough to take advantage of that option. Therefore, the prognosis of HCC patients remains extremely poor, with a 5 -year survival rate of only $5 \%$ (3). Chemotherapy is often used in conjunction with surgery and radiation (4). However, HCC is a type of cancer with high resistance to conventional anticancer agents. Paclitaxel is one of the most effective anticancer drugs discovered in the past few decades. For a number of years it has been clinically used in the treatment of various cancers, including HCC (5). However, chemoresistance due to paclitaxel-induced nuclear factor $\kappa \mathrm{B}(\mathrm{NF}-\kappa \mathrm{B})$ activation is an important cause of suboptimal therapeutic effect (6). Therefore, the search for new effective chemopreventive and chemotherapeutic agents with the ability of directly inhibiting HCC cell growth or enhancing the anticancer activity of conventional chemotherapeutic agents is urgent.

Accumulating data suggest that a number of signaling elements, such as NF- $\mathrm{B}$ (7), TLR-4 (8), Ras (9) and Akt (10), not only promote cancer progression but also confer chemoresistance. Among these signaling elements, NF- $\mathrm{\kappa B}$ is one of the most investigated transcription factors, the activation of which has been found to control multiple cellular processes in cancer. Currently, many researchers have reported that NF- $\mathrm{kB}$ activation plays a facilitating role in cell cycle progression and apoptotic resistance. Additionally, chemotherapy-induced activation of NF- $\mathrm{KB}$ may blunt the ability of chemotherapy itself 


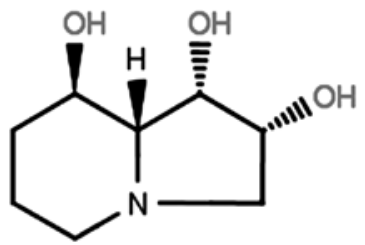

Figure 1. Chemical structure of swainsonine.

$(11,12)$. Therefore, the inhibition of NF- $\mathrm{B}$ may be an effective treatment strategy for inhibiting tumor growth and progression as well as in restoring the sensitivity of cancer cells to cytotoxic drugs (13).

With the above findings in mind, we speculated that new strategies with $\mathrm{NF}-\kappa \mathrm{B}$ suppression activity, which directly inhibits cancer cell growth and restores the sensitivity of cancer cells to the number of cytotoxic drugs (such as paclitaxel), may be an effective method for treating HCC. Swainsonine, a new class of compounds that display a wide range of pharmacological effects, has been under extensive research to examine its potential anticancer and therapeutic biological activities. A number of swainsonine-related studies confirmed that swainsonine may inhibit growth and induce apoptosis in tumor cells (14-16). However, the detailed mechanism underlying the use of swainsonine as an anticancer drug, especially through inhibition of NK- $\mathrm{NB}$ activation, remains unknown. Therefore, an in-depth study is required. Nevertheless, research reports regarding anti-HCC activity and related mechanisms remain scarce. To determine the direct anti-HCC effect of swainsonine, the underlying molecular mechanisms and whether swainsonine sensitizes HCC cells to paclitaxel, we evaluated the effectiveness of swainsonine alone or in combination with paclitaxel in vitro and in vivo. To the best of our knowledge, our results provide for the first time evidence that swainsonine is a reliable candidate for chemotherapeutic treatment of HCC that should be further investigated.

\section{Materials and methods}

Sample preparation. Endotoxin-free synthetic swainsonine (purity over 99\%) was supplied by Sigma (St. Louis, MO, USA). The structure of this compound is shown in Fig. 1. Swainsonine was prepared in $\mathrm{Ca}^{2+}$-free and $\mathrm{Mg}^{2+}$-free phosphate-buffered saline (PBS), sterilized by ultrafiltration and stored at $-20^{\circ} \mathrm{C}$ until diluted to the final concentration in fresh medium before each experiment.

Cell lines and cell culture. Human hepatoma HepG2, SMCC7721, Huh7 and MHCC97-H cells and human hepatocyte HL-7702 cells (maintained in our laboratory, originally obtained from the Cell Bank of the Type Culture Collection of Chinese Academy of Sciences) were cultured in Dulbecco's modified Eagle's medium (DMEM) supplemented with 10\% fetal bovine serum (FBS) (Invitrogen, Carlsbad, CA, USA), penicillin $(100 \mathrm{IU} / \mathrm{ml})$, streptomycin $(100 \mu \mathrm{g} / \mathrm{ml})$ and trypsin (400 IU/l). These were cultured at $37^{\circ} \mathrm{C}$ in $5 \% \mathrm{CO}_{2}$ and $95 \%$ humidified air. The medium was changed every 2 days. After reaching $\sim 60-70 \%$ confluence, the cells were treated with different concentrations of swainsonine as indicated in Results.
MTT assay for cytotoxicity. A 3-(4,5-dimethylthiazol-2-yl)2,5-diphenyltetrazolium bromide (MTT) assay was performed to determine the number of viable cells. The cells were cultured in 96-well plates at a density of $4 \times 10^{3}$ cells/well with fresh medium containing swainsonine at the indicated concentrations $(0.06,0.12,0.24,0.48$ and $0.96 \mu \mathrm{g} / \mathrm{ml})$. After treatment for 24,48 or $72 \mathrm{~h}$, MTT solution was added to each well $(5 \mathrm{mg} / \mathrm{ml})$ and incubated at $37^{\circ} \mathrm{C}$ for $4 \mathrm{~h}$. The MTT-formazan product dissolved in dimethyl sulfoxide (DMSO) was measured at a wavelength of $490 \mathrm{~nm}$ with a microplate reader (Bio-Rad Laboratories, Hercules, CA, USA). The reduction in the viability of the swainsonine-treated cells was expressed as the percentage compared with swainsonine-free control cells.

Ki-67 expression analysis for the cellular growth. Flow cytometric analysis was performed for Ki-67 studies as previously described (17). Briefly, following incubation with 0.00, 0.09, 0.17 and $0.33 \mu \mathrm{g} / \mathrm{ml}$ of swainsonine for $24 \mathrm{~h}$, MHCC97-H cells treated with or without swainsonine were harvested, fixed in $70 \%$ (v/v) ethanol, washed and incubated for $20 \mathrm{~min}$ with $1 \%$ bovine serum albumin (BSA). Then, the cells were incubated with 1:200 diluted anti-Ki-67 (FITC-conjugated; Bioscience, Beijing, China). Data were obtained and analyzed by flow cytometry (Becton-Dickinson, San Jose, CA, USA).

Effects of swainsonine on cell cycle progression and apoptosis. To explore whether the growth inhibitory effect of swainsonine was caused by cell cycle arrest and apoptosis, cell cycle distribution and Annexin V analyses were utilized.

Cell cycle distribution analysis. Following incubation with $0.00,0.09,0.17$ and $0.33 \mu \mathrm{g} / \mathrm{ml}$ of swainsonine for $24 \mathrm{~h}$, MHCC97-H cells treated with or without swainsonine were harvested by trypsinization and fixed with ice-cold $70 \%$ ethanol for $48 \mathrm{~h}$ at $4^{\circ} \mathrm{C}$. After being rinsed twice with PBS, the cells were treated with RNase A at $1 \mathrm{mg} / \mathrm{ml}$ for $30 \mathrm{~min}$ at $37^{\circ} \mathrm{C}$. After staining with $40 \mu \mathrm{l}$ of $0.1 \mathrm{mg} / \mathrm{l}$ propidium iodide (PI), stained cells were subjected to flow cytometric analysis.

Western blot analysis for cell cycle regulatory proteins. The cells were processed for protein extraction and western blot analysis as previously described (17). The primary antibodies were anti-cyclin D1 (diluted 1:500, rabbit polyclonal), anti-cyclin E (diluted 1:300, mouse monoclonal), anti-cyclindependent kinase (Cdk) 2 (diluted 1:300, mouse monoclonal), anti-Cdk 4 (diluted 1:300, mouse monoclonal), anti-p21 (diluted 1:200, mouse monoclonal), anti-p27 (diluted 1:200, mouse monoclonal) (all from Santa Cruz Biotechnology, Santa Cruz, CA, USA) and anti-glyceraldehyde-3-phosphate dehydrogenase (GAPDH) (diluted 1:400, rabbit monoclonal C-2; Santa Cruz Biotechnology).

Apoptotic cell determination. An Annexin V-FITC/PI apoptosis detection kit (Annexin V-FITC/PI staining kit; Immunotech Co., Marseille, France) was used for the detection of cell apoptosis. In brief, $1.5 \times 10^{5}$ cells were plated in 24-well plates and treated with $0.00,0.09,0.17$ and $0.33 \mu \mathrm{g} / \mathrm{ml}$ of swainsonine for $24 \mathrm{~h}$. The cells treated with or without swainsonine were collected, washed in cold PBS, incubated for 15 min with fluorescein-conjugated Annexin V and PI 
according to the manufacturer's instructions and analyzed by flow cytometry.

Morphological evaluation of apoptotic MHCC97-H cells. The cells were treated with swainsonine for $24 \mathrm{~h}$ at concentrations of 0.00 (PBS, vehicle as control) and $0.33 \mu \mathrm{g} / \mathrm{ml}$. Morphological changes were observed with an inverted microscope (Olympus Corp., Tokyo, Japan). The Hoechst 33342 staining technique was used to analyze the alterations in the nuclear morphology of cells. Different interfered cells were washed in PBS and fixed in $70 \%$ ethanol for $2 \mathrm{~h}$ at $4^{\circ} \mathrm{C}$. Cell nuclei were stained with $5 \mu \mathrm{g} / \mathrm{ml}$ Hoechst 33342 (Sigma). After staining with Hoechst 33342, the changes in nuclear morphology were visualized by fluorescence microscopy (Olympus Corp.). The ultrastructure morphological change was evaluated by transmission electron microscopy (TEM) as described below. The cells were collected and fixed in $2.5 \%$ glutaraldehyde, precooled at $4^{\circ} \mathrm{C}$ and stored at $4^{\circ} \mathrm{C}$ overnight. Then the cells were dehydrated, embedded, cut into ultrathin sections and stained routinely. The stained sections were scanned with an electron microscope (JEM-2000EX, JEOL Ltd., Tokyo, Japan) for ultrastructure observations.

Western blot analysis of apoptosis regulatory proteins. Apoptosis-related gene fusion proteins were identified by western blot analysis. The specific primary antibodies were anti-Bax (diluted 1:300, mouse monoclonal), anti-Bcl-2 (diluted 1:300, mouse monoclonal), anti-Fas (diluted 1:300, mouse monoclonal) and anti-Fas-L (diluted 1:300, rabbit polyclonal) (all from Santa Cruz Biotechnology).

Western blot analysis for the effects of swainsonine on the activation of $\mathrm{NF}-\kappa \mathrm{B}$ in $\mathrm{MHCC} 97-\mathrm{H}$ cells. The preparation of cytoplasmic and nuclear extracts was performed using the Nuclear Extract kit (HyClone-Pierce, South Logan, UT, USA) according to the manufacturer's instructions. To measure nuclear NF- $\kappa \mathrm{B} / \mathrm{p} 65$ and cytoplasmic NF- $\mathrm{NB} / \mathrm{p} 65$ expression, western blot analysis was performed. Related fusion proteins were identified using anti-NF- $\mathrm{B} / \mathrm{p} 65$ (diluted 1:500, rabbit monoclonal; Cell Signaling Technology, Beverly, MA, USA) primary antibody.

Effects of swainsonine on chemosensitization of HCC for paclitaxel toxicity. To confirm the sensitization to paclitaxel toxicity from swainsonine in vitro, MHCC97-H cells were treated with swainsonine $(0.03 \mu \mathrm{g} / \mathrm{ml})$, paclitaxel $(10 \mathrm{ng} / \mathrm{ml}$; Beijing Sihuan Pharmaceutical Co., Ltd., Beijing, China) or swainsonine $(0.03 \mu \mathrm{g} / \mathrm{ml})$ plus paclitaxel $(10 \mathrm{ng} / \mathrm{ml})$ for $24 \mathrm{~h}$ and the effect on growth inhibition was examined using the cell viability assay described above. In vivo, female athymic nude mice (6-8 weeks old) were maintained in pathogenlimited conditions at the animal resources center at the Fourth Military Medical University. The Local Animal Care and Use Committee of the Fourth Military Medical University approved all animal experimental procedures. MHCC97-H cells $\left(1 \times 10^{7}\right.$ in $0.1 \mathrm{ml}$ of serum-free growth medium) were inoculated s.c. on the left side of the armpit. The injection procedure took 2-3 min; all mice recovered in $<5$ min without showing signs of stress or casualty. All mice were monitored for 5 min after cell administration and were returned to their cages. Two weeks after tumor cell implantation, 40 tumor-bearing mice were randomly divided into four groups and received one of the following treatments by a single i.p. injection into the lower right quadrant of the peritoneum. One group of mice $(\mathrm{n}=10)$ was treated with swainsonine $(1 \mathrm{mg} / \mathrm{kg})$ thrice weekly plus paclitaxel $(5 \mathrm{mg} / \mathrm{kg})$ weekly for 4 weeks. A second group of mice $(n=10)$ was treated with swainsonine alone $(1 \mathrm{mg} / \mathrm{kg})$ thrice weekly for 4 weeks. A third group $(n=10)$ was treated with paclitaxel alone $(5 \mathrm{mg} / \mathrm{kg})$ weekly for 4 weeks. The control group $(n=10)$ received normal saline. Tumor size was measured twice weekly. Tumor volume (V) was calculated using the following formula: $\mathrm{V}=(\mathrm{a} \times \mathrm{b} \times \mathrm{c}) / 2$, are $\mathrm{a}$ and $\mathrm{b}$ were the shorter and longer diameters of each tumor, respectively and $\mathrm{c}$ was the thickness. At the end of the experiment, mice underwent euthanasia with $\mathrm{CO}_{2}$. The tumors of each group were harvested to profile the gene expression associated with growth (Ki-67; Santa Cruz Biotecnhology) and apoptosis (Bax and Bcl-2; Santa Cruz Biotecnhology) by western blot analysis. To identify whether swainsonine chemosensitizes HCC to paclitaxel-induced cytotoxicity via attenuating the constitutive activation of $\mathrm{NF}-\kappa \mathrm{B}$, we also examined its cellular localization in paclitaxel (alone or in combination with swainsonine)treated MHCC97-H cells.

Statistical analysis. The significance of differences between the groups was determined with one-way ANOVA (SPSS10.0 statistical software). The results with a P-value $\leq 0.05$ were considered statistically significant. Data are expressed as the mean \pm standard error of the mean (SEM) of separate experiments ( $n \geq 3$, where $\mathrm{n}$ represents the number of independent experiments).

\section{Results}

Cytotoxicity of swainsonine in both human hepatoma and HL-7702 cells. The MTT experiment confirmed that human hepatoma cells were sensitive to swainsonine and that swainsonine inhibited the growth of human hepatoma cells in a dose- and time-dependent manner. As shown in Fig. 2, exposure of hepatoma cells to different concentrations of swainsonine $(0.06-0.96 \mu \mathrm{g} / \mathrm{ml})$ at different time points resulted in a statistically significant change in cell viability $(n=3, P<0.05)$. The $\mathrm{IC}_{50}$ value at $24 \mathrm{~h}$ post-treatment with swainsonine for HepG2, SMCC7721, Huh7 and MHCC97-H cells was 0.43, 0.41, 0.39 and $0.33 \mu \mathrm{g} / \mathrm{ml}$, respectively. However, different concentrations of swainsonine had no significant toxic effects on hepatocytes for the different time periods $(n=3, P>0.05)$. Therefore, swainsonine reduced the viability of hepatoma cells but was only slightly toxic to HL-7702 hepatocyte cells. Taking into account the more aggressive and highly metastatic nature of HCC, MHCC97-H cells were then selected as a model system with which to conduct mechanistic studies in vitro and in vivo.

Swainsonine inhibits the growth of MHCC97-H cells. The effects of swainsonine on the growth of MHCC97-H cells were examined through the analysis of Ki-67 expression (Fig. 3A and B). Our data demonstrated that a considerable decrease in the level of expression of $\mathrm{Ki}-67$ occurred in a dosedependent manner after $24 \mathrm{~h}$ of swainsonine treatment. These outcomes suggest that treatment with swainsonine results in the inhibition of cell growth of MHCC97-H cells. 
HepG2

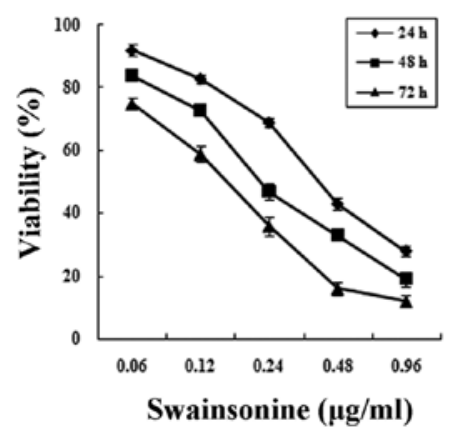

Huh7

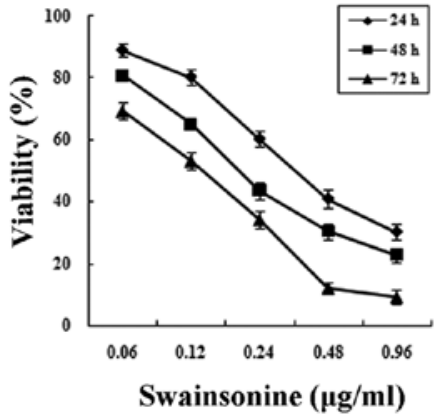

SMCC7721

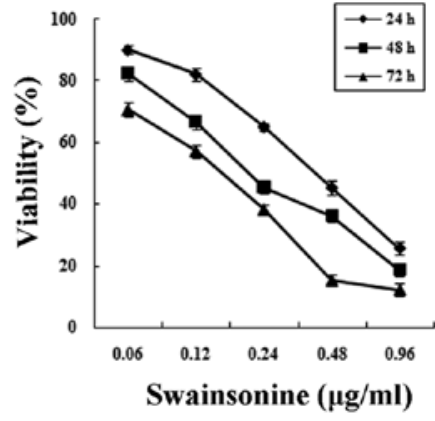

MHCC97-H

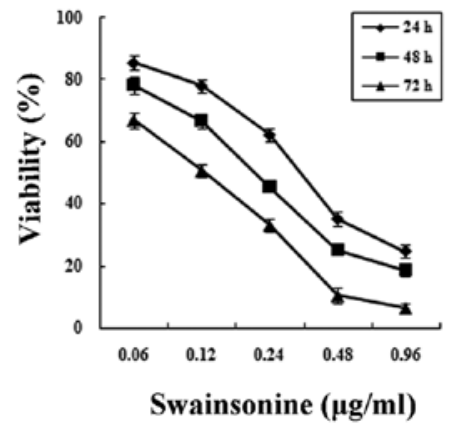

HL-7702

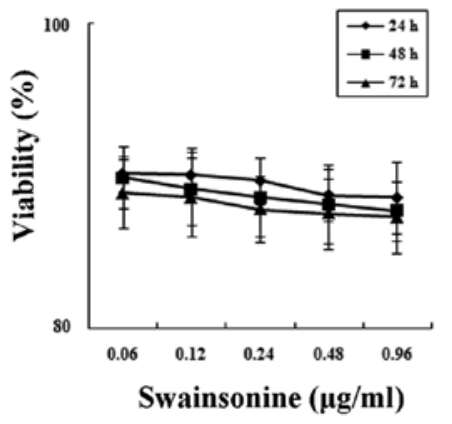

Figure 2. Dose- and time-dependent effects of swainsonine on the viability of hepatoma and hepatocyte cells. Cells were incubated with $0.06-0.96 \mu \mathrm{g} / \mathrm{ml}$ of swainsonine for 24,48 and $72 \mathrm{~h}$ and cell growth was determined by MTT assay.
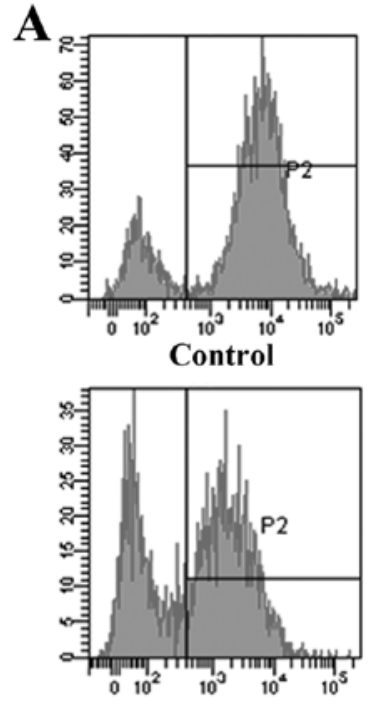

Swainsonine $0.17 \mu \mathrm{g} / \mathrm{m}$
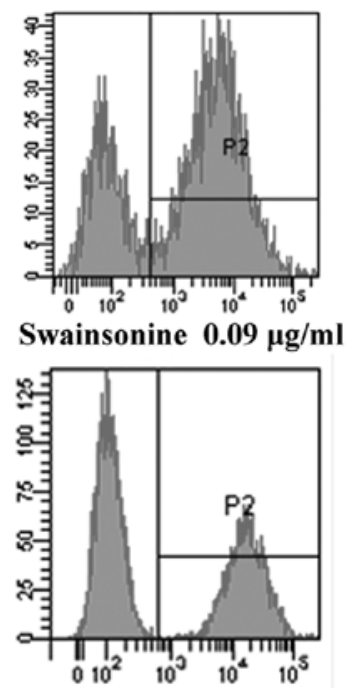

Swainsonine $0.33 \mu \mathrm{g} / \mathrm{ml}$
B

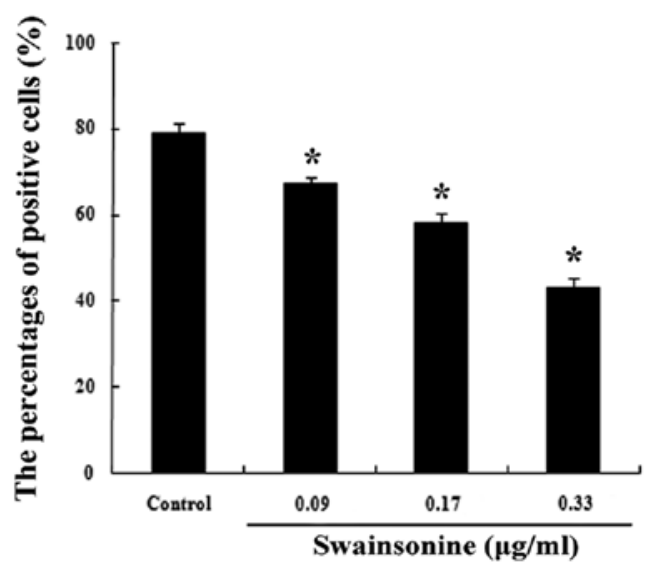

Figure 3. Effects of swainsonine on the growth of MHCC97-H cells. (A) Treatment with different concentrations of swainsonine resulted in the decreased growth of MHCC97-H cells, as determined by Ki-67 analysis. (B) The expression of Ki-67 in each group of cells is reflected in the column chart. "P<0.05 compared to the control.

Effects of swainsonine on cell cycle progression and apoptosis Swainsonine induces cell cycle arrest in MHCC97-H cells. Dose-response analysis was performed, whereby MHCC97-H cells were exposed to increased concentrations of swainsonine for 24 h. As shown in Fig. 4 and Table I, following incubation with different concentrations of swainsonine, the proportion of cells in the G0/G1 phase increased substantially compared to the control group; while the number of cells in the $\mathrm{S}$ and
G2/M phase were reduced to some extent within $24 \mathrm{~h}$ ( $\mathrm{n}=3$, $\mathrm{P}<0.05$ ), suggesting $\mathrm{G} 0 / \mathrm{G} 1$ phase arrest. Our data suggest that swainsonine inhibits MHCC97-H cell growth by blocking the G0/G1 to $S$ phase transition in the cell cycle in a dosedependent manner.

Swainsonine alters the expression of cell cycle-associated proteins. Cyclins (D1 and E), Cdk2, Cdk4 and Cdk inhibitors, 


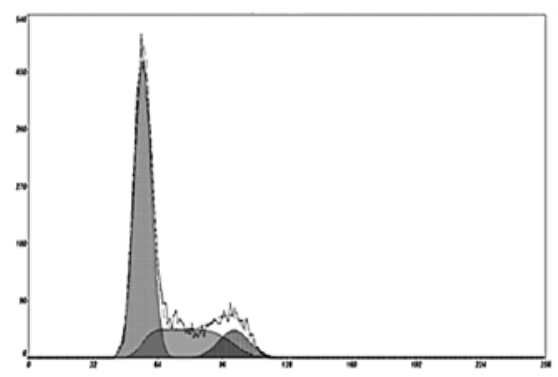

Control

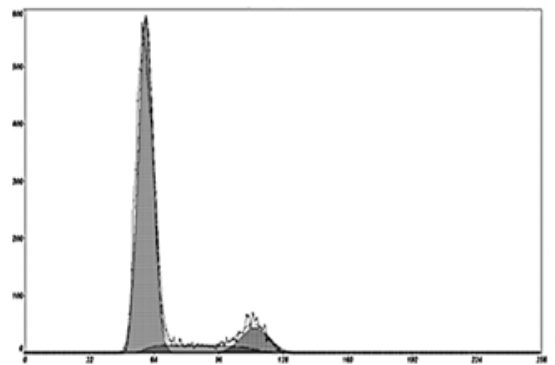

Swainsonine $0.17 \mu \mathrm{g} / \mathrm{ml}$

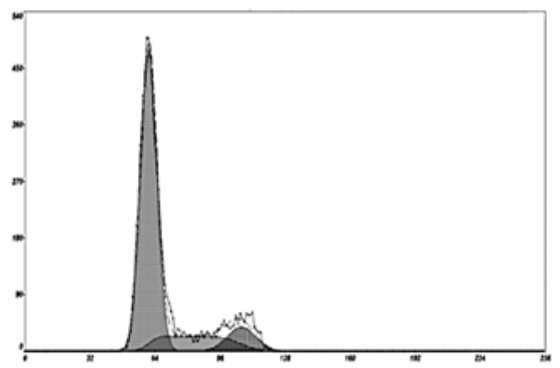

Swainsonine $0.09 \mu \mathrm{g} / \mathrm{ml}$

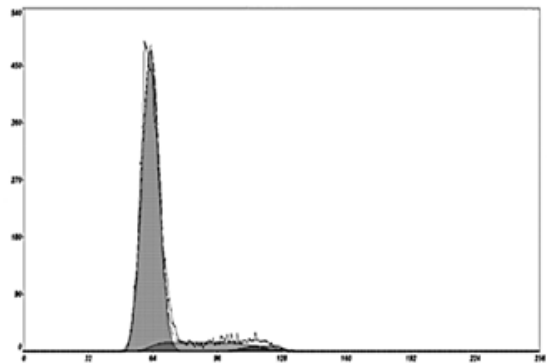

Swainsonine $0.33 \mu \mathrm{g} / \mathrm{ml}$

Figure 4. DNA levels in each treatment group. Cells were treated with different concentrations of swainsonine for $24 \mathrm{~h}$ and then analyzed using flow cytometry. Flow cytometric histograms are representative of 3 separate experiments.

Table I. Effects of swainsonine on cell cycle distribution (\%).

\begin{tabular}{lccc}
\hline & G0/G1 & $\mathrm{S}$ & G2/M \\
\hline Control & $62.77 \pm 2.06$ & $25.68 \pm 2.12$ & $11.55 \pm 1.83$ \\
Swainsonine $(\mu \mathrm{g} / \mathrm{ml})$ & & & \\
0.09 & $73.24 \pm 1.76^{\mathrm{a}}$ & $16.73 \pm 1.73^{\mathrm{a}}$ & $10.03 \pm 1.68$ \\
0.17 & $78.58 \pm 2.06^{\mathrm{a}}$ & $11.40 \pm 1.80^{\mathrm{a}}$ & $10.02 \pm 1.74$ \\
0.33 & $84.04 \pm 2.28^{\mathrm{a}}$ & $8.21 \pm 1.17^{\mathrm{a}}$ & $7.75 \pm 1.29^{\mathrm{a}}$ \\
\hline
\end{tabular}

${ }^{\mathrm{a}} \mathrm{P}<0.05$ compared to the control.

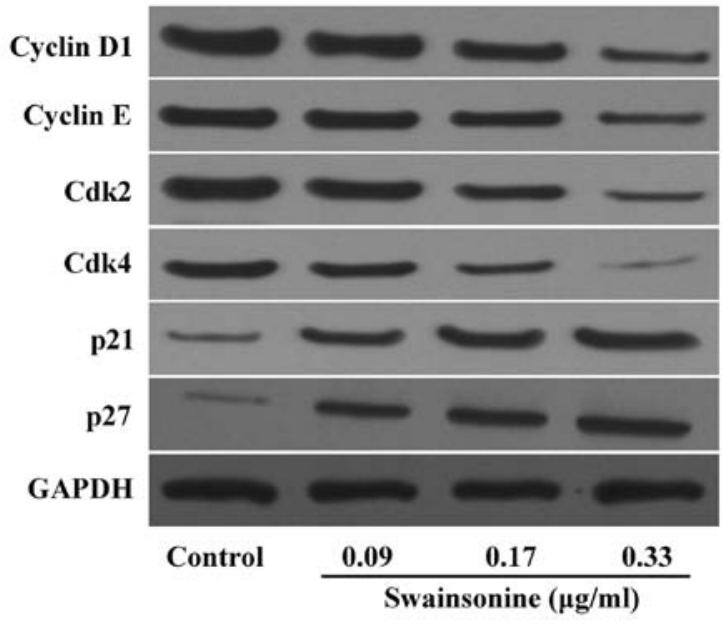

Figure 5. Swainsonine treatment leads to altered expression of cell cyclerelated proteins in human hepatoma cells. Western blot analysis of cyclin D1, cyclin E, Cdk2, Cdk4, p21 and p27 protein levels in cell lysates from MHCC97-H cells treated with the indicated concentration of swainsonine. GAPDH was used as the loading control. p21 and p27 are important for cell growth and are key signaling proteins in cell cycle progression. We investigated whether swainsonine blocks the G0/G1 to $S$ phase transition through altering the expression of these proteins. As shown in Fig. 5, swainsonine induced a dose-dependent decrease in cyclin D1, cyclin E, Cdk2 and Cdk4 protein levels, while an increased expression of p21 and p27 was observed after swainsonine treatment. Taken together, these results indicate that swainsonine decreased growth by blocking cell cycle progression via the upregulation of p21 and p27 and/or the downregulation of cyclin D1, cyclin E, Cdk2 and Cdk4 expression.

Swainsonine induces apoptosis in MHCC97-H cells. We assessed the apoptotic rate of the cells treated with different concentrations of swainsonine for $24 \mathrm{~h}$ using the Annexin V/PI staining assay. The results (Fig. 6 and Table II) indicated that after swainsonine treatment for $24 \mathrm{~h}$, viable cells decreased clearly and the proportion of early and late apoptotic cells increased $(n=3, P<0.05)$ in a dose-dependent manner. These 
Table II. Effects of swainsonine on cell apoptosis (\%).

\begin{tabular}{lcccr}
\hline & Normal & Early apoptosis & Late apoptosis & Necrosis \\
\hline Control & $97.88 \pm 1.19$ & $1.62 \pm 0.06$ & $0.40 \pm 0.03$ & $0.01 \pm 0.00$ \\
Swainsonine $(\mu \mathrm{g} / \mathrm{ml})$ & & & \\
0.09 & $79.36 \pm 2.03^{\mathrm{a}}$ & $13.30 \pm 1.57^{\mathrm{a}}$ & $4.74 \pm 0.29^{\mathrm{a}}$ & $2.60 \pm 0.11^{\mathrm{a}}$ \\
0.17 & $64.88 \pm 1.77^{\mathrm{a}}$ & $21.19 \pm 1.00^{\mathrm{a}}$ & $12.72 \pm 0.51^{\mathrm{a}}$ & $1.21 \pm 0.06^{\mathrm{a}}$ \\
0.33 & $50.35 \pm 1.56^{\mathrm{a}}$ & $27.61 \pm 1.03^{\mathrm{a}}$ & $20.96 \pm 0.97^{\mathrm{a}}$ & $1.08 \pm 0.02^{\mathrm{a}}$ \\
\hline
\end{tabular}

${ }^{\mathrm{a}} \mathrm{P}<0.05$ compared to the control.

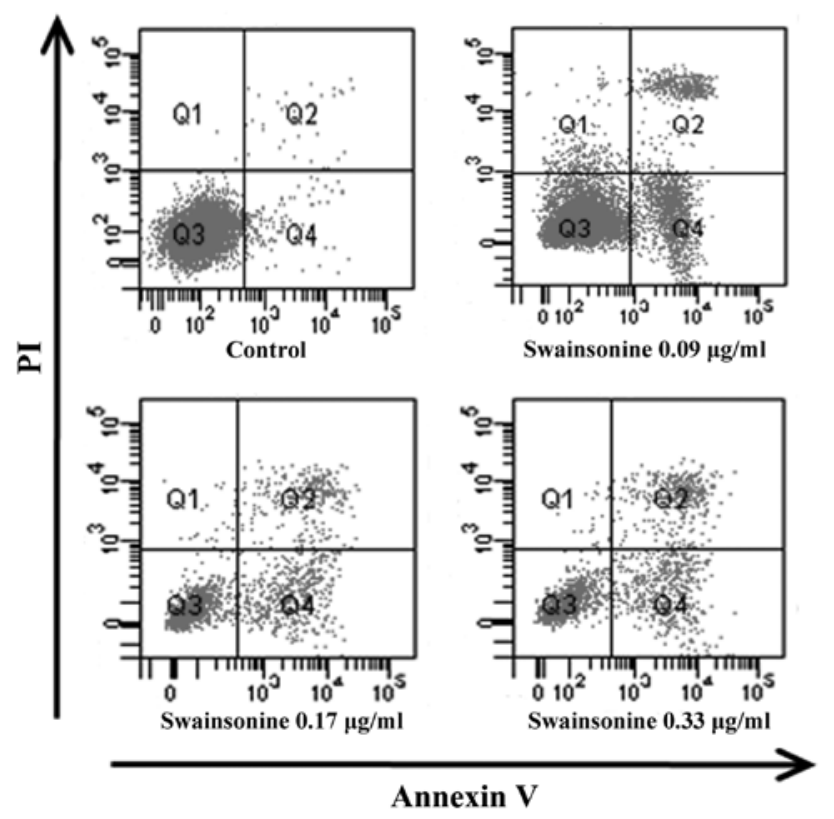

Figure 6. Effects of swainsonine on the apoptosis of MHCC97-H cells. Cells were treated with different concentrations of swainsonine for $24 \mathrm{~h}$ and then analyzed for apoptosis by flow cytometry for Annexin V and PI staining. Swainsonine significantly induced apoptosis in MHCC97-H cells.

results showed that swainsonine significantly increased apoptosis.

Swainsonine induces apoptotic morphological changes in MHCC97-H cells. Morphological analyses showed that MHCC97-H cells underwent marked morphological changes after incubation with swainsonine. Bright field observations revealed that cells treated with swainsonine $(0.33 \mu \mathrm{g} / \mathrm{ml})$ became round, shrunken and detached from the surface of the flask (Fig. 7A). Subsequently, Hoechst 33342 staining also revealed characteristic morphological features, for example, the cells shrank, became circular, intensely fluorescent, fragmented and had condensed nuclei when treated with swainsonine (Fig. 7B). The apoptotic features of cells were further confirmed by electron microscopy. Cells were smaller in size, lost microvilli, had irregular cell outlines, had condensed, fractured and marginalized chromatin and had increased numbers of lysosomes (Fig. 7C). The morphological changes observed were consistent with changes associated with apoptosis.

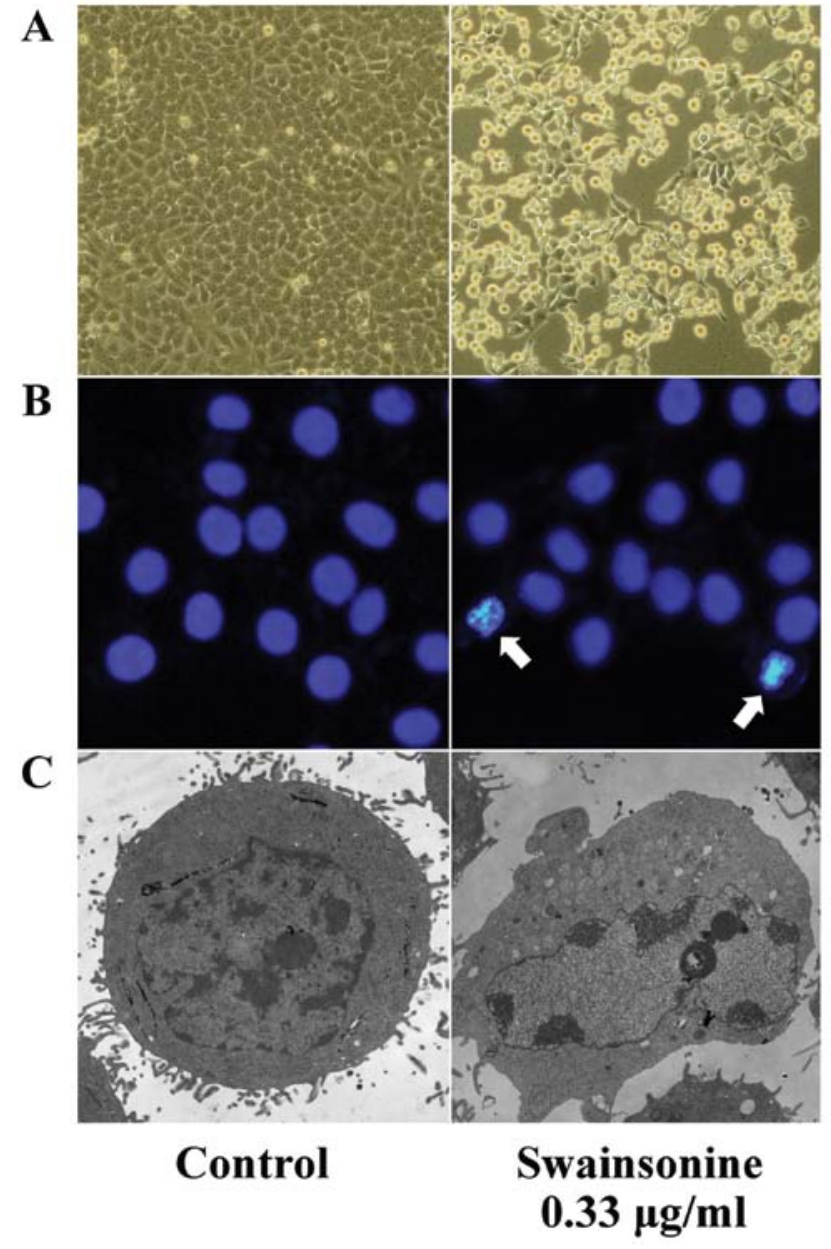

Figure 7. Morphological alterations of MHCC97-H cells treated with swainsonine for $24 \mathrm{~h}$. (A) Following treatment, a significant change in cell morphology was observed in MHCC97-H cells as examined under a phasecontrast microscope. Original magnification, x100.(B) The cells were stained with Hoechst 33342 and the apoptotic morphological changes in the nuclear region were captured by fluorescence microscopy. Original magnification, $\mathrm{x} 400$. The arrows in the image indicate the apoptotic morphological changes in the nuclear region. (C) TEM shows the fine cellular structures of each treatment group. Original magnification, x10,000.

Swainsonine alters the expression of apoptosis-related proteins. To further clarify the apoptotic mechanisms of human hepatoma cells induced by swainsonine treatment, we analyzed the proteins of MHCC97-H cells treated with different concentrations of swainsonine using western blot 


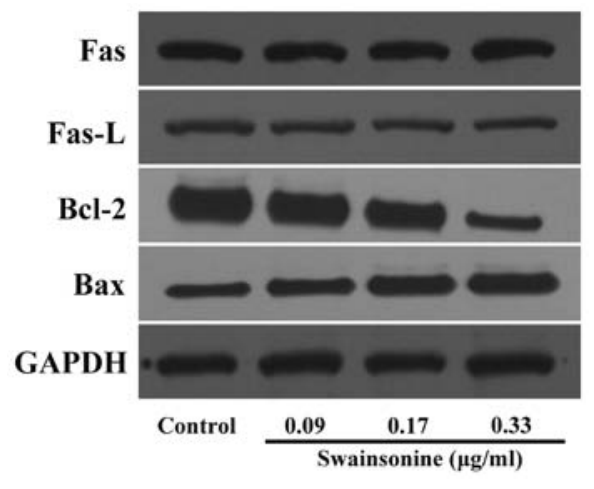

Figure 8. Expression of apoptosis-related proteins in MHCC97-H cells treated with swainsonine. The protein was extracted and the expression of apoptosis-related proteins was analyzed by western blot assay.

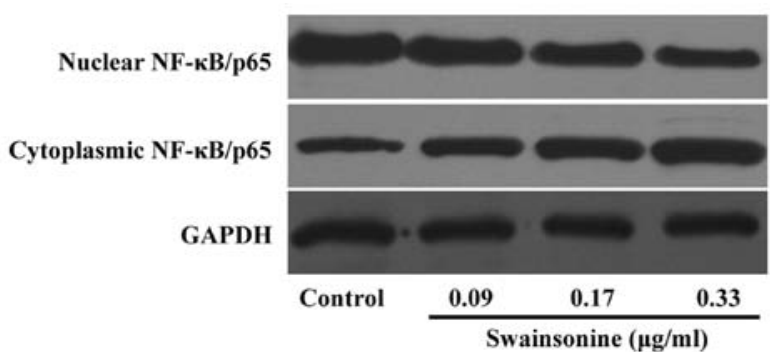

Figure 9. Swainsonine attenuates constitutive $\mathrm{NF}-\kappa \mathrm{B}$ activation by inhibiting the nuclear translocation of $\mathrm{NF}-\kappa \mathrm{B} / \mathrm{p} 65$ in MHCC97-H cells. Nuclear and cytoplasmic extracts were prepared from cells treated with swainsonine $(0.00,0.09,0.17$ and $0.33 \mu \mathrm{g} / \mathrm{ml})$ for $24 \mathrm{~h}$ and the expression of $\mathrm{NF}-\kappa \mathrm{B} / \mathrm{p} 65$ was determined by western blot analysis.

assay. We found that the expression levels of Fas and Fas-L were not changed significantly $(n=3, P>0.05)$. Furthermore, we observed a dose-dependent reduction in the levels of the anti-apoptotic protein $\mathrm{Bcl}-2$, whereas a concomitant increase in the level of pro-apoptotic protein Bax was observed (Fig. 8) $(n=3, P<0.05)$. The results indicate that swainsonine induces apoptosis in MHCC97-H cells mainly through a mitochondriamediated internal pathway.

Swainsonine attenuates the constitutive activation of $N F-\kappa B$ in MHCC97-H cells. Since NF- $\mathrm{BB}$ activation controls the expression of a number of genes involved in cell growth and survival through direct and indirect mechanisms, we investigated whether the treatment of HCC cells with swainsonine has an impact on NF- $\mathrm{NB}$ activation in HCC cells. We revealed that swainsonine treatment caused a marked and dose-dependent increase in NF- $\kappa \mathrm{B}$ levels in the cytoplasmic fraction of MHCC97-H cells with a simultaneous decrease in the nuclear fraction (Fig. 9). The results indicate that swainsonine suppresses constitutive activation of $\mathrm{NF}-\kappa \mathrm{B}$ in $\mathrm{HCC}$ cells.

Swainsonine chemosensitizes HCC to paclitaxel-induced cytotoxicity. Since chemoresistance, due to paclitaxel-induced $\mathrm{NF}-\kappa \mathrm{B}$ activation, is an important cause of a suboptimal therapeutic effect we investigated whether swainsonine acts as a chemosensitizer in HCC in vitro and in vivo. At a concentration of $0.03 \mu \mathrm{g} / \mathrm{ml}$, swainsonine did not significantly inhibit the growth of the MHCC97-H cell line after $24 \mathrm{~h}$ of treatment (Fig. 10A). We performed further cell viability analysis in the MHCC97-H cells incubated with paclitaxel alone or swainsonine plus paclitaxel. As noted in Fig. 10A, paclitaxel treatment alone inhibited the grow th of MHCC97-H cells, while combined treatment with swainsonine led to a significant enhancement in the inhibitory effects of paclitaxel. This suggests that swainsonine at a concentration of $0.03 \mu \mathrm{g} / \mathrm{ml}$ does not trigger apoptosis alone, but is capable of sensitizing a cell response to paclitaxel. Furthermore, the in vivo antitumor activity of the paclitaxel/ swainsonine combination was evaluated in nude mice xenografts. The optimum doses of paclitaxel and swainsonine used in this study were adopted according to our preliminary experiments (data not shown). We found that paclitaxel alone inhibited the growth of established tumors, while combined treatment of paclitaxel plus swainsonine resulted in a more significant suppression of established tumors compared to paclitaxel or swainsonine alone $(\mathrm{P}<0.05)$. Swainsonine alone at low concentrations was unable to significantly inhibit tumor growth in the xenograft-bearing mice (Fig. 10B and C). We further analyzed the growth- and apoptosis-related molecules of tumors in each group. Importantly, we found that tumors treated with paclitaxel plus swainsonine showed lower Ki-67 and $\mathrm{Bcl}-2$, with higher Bax levels in comparison to tumors treated alone with paclitaxel or swainsonine (Fig. 10D) $(\mathrm{P}<0.05)$. Our data demonstrated that treatment with swainsonine increased the efficacy of the paclitaxel-induced inhibition of tumor xenografts in mice. In addition, we also determined the cellular localization of NF- $\mathrm{NB}$ in paclitaxel (alone or in combination with swainsonine)-treated MHCC97-H cells. Our data showed that paclitaxel induced an enhanced accumulation of NF- $\kappa \mathrm{B}$ in a nuclear compartment and a concomitant decrease in cytoplasmic fraction in a dose-dependent manner (Fig. 10E). However, swainsonine was effective in inhibiting this paclitaxel-induced activation of NF- $\mathrm{NB}$ in $\mathrm{MHCC} 97-\mathrm{H}$ cells (Fig. 10F). Our data indicate that swainsonine may be an important chemotherapeutic agent for HCC and that it potentiates the anticancer efficacy of paclitaxel by acting as a chemosensitizer.

\section{Discussion}

Despite many years of intensive research, HCC remains a devastating malignancy due to the lack of effective therapy. Systemic chemotherapy is the only remaining option, especially for patients with inoperable or metastatic disease. Although systemic drugs have been tested for patients with $\mathrm{HCC}$, the prognosis of these patients is not yet favorable. Most patients with advanced HCC are destined to develop resistance to conventional anticancer agents. Therefore, the development of new drugs with the ability to directly inhibit cancer cell growth or potentiate the cytotoxic effect of other agents for the treatment of HCC is required (18). Recently, chemotherapy and other intervention strategies using naturally occurring agents have emerged as a promising alternative option to improve the quality of life for HCC patients (19).

Swainsonine, an extract from Astragalus membranaceus, is a known potent and specific inhibitor of lysosomal acid and Golgi $\alpha$-mannosidase II (20). Golgi $\alpha$-mannosidase II is an important member of the N-glycosylation pathway, which 

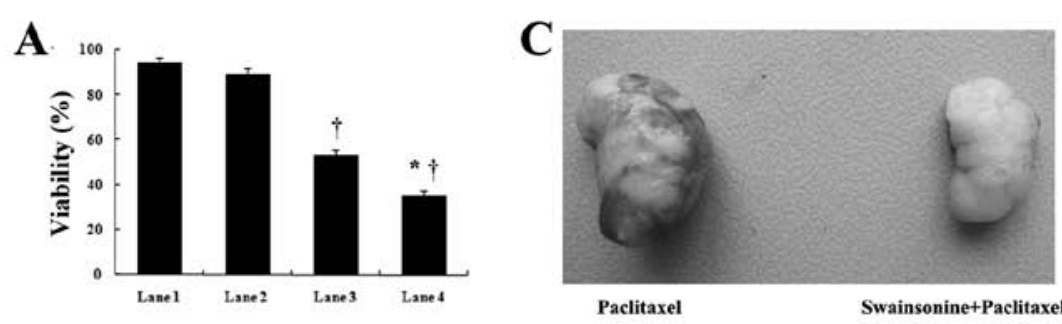

D
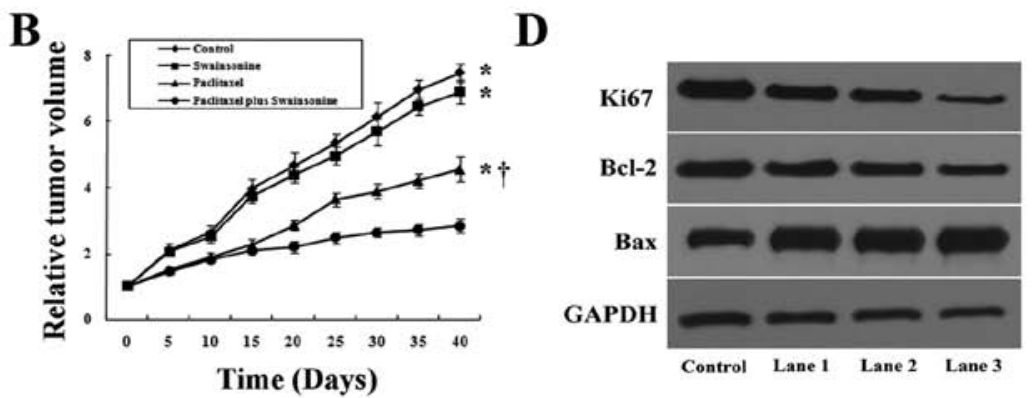

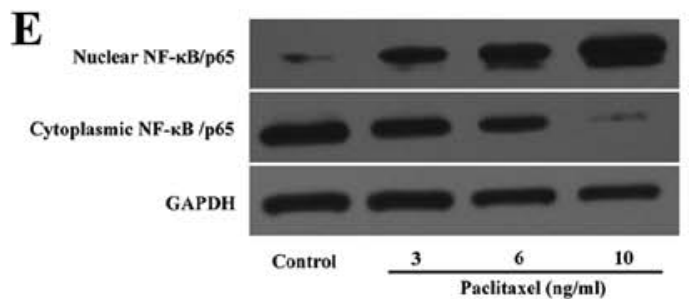

F

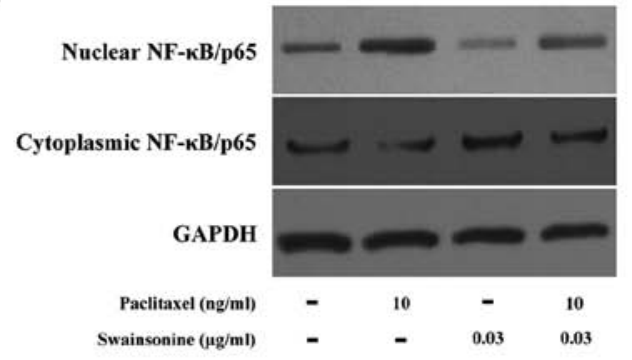

Figure 10. Swainsonine chemosensitizes MHCC97-H cells to paclitaxel treatment in vitro and in vivo. (A) MHCC97-H cells were treated with swainsonine $(0.03 \mu \mathrm{g} / \mathrm{ml})$, paclitaxel $(10 \mathrm{ng} / \mathrm{ml})$ or swainsonine $(0.03 \mu \mathrm{g} / \mathrm{ml})$ plus paclitaxel $(10 \mathrm{ng} / \mathrm{ml})$ for $24 \mathrm{~h}$. Percent viability was measured by MTT assay. Lane 1 , control; lane 2, cells treated with swainsonine; lane 3 , cells treated with paclitaxel; lane 4 , cells treated with swainsonine plus paclitaxel. ${ }^{*} \mathrm{P}<0.05$ compared to paclitaxel alone. ${ }^{\dagger} \mathrm{P}<0.05$ compared to the control. (B) In vivo anticancer activity assay. Drug treatment groups received paclitaxel, swainsonine or paclitaxel and swainsonine. The tumor volumes were measured on the indicated day. ${ }^{*} \mathrm{P}<0.05$ vs. paclitaxel plus swainsonine group. ${ }^{\dagger} \mathrm{P}<0.05$ compared to the control. $(\mathrm{C})$ At the end of the experiment, representative tumors were collected. (D) The expression levels of Bax, Bcl-2 and Ki-67 under in vivo conditions. Lane 1, control; lane 2, mice treated with swainsonine; lane 3, mice treated with paclitaxel; lane 4, mice treated with swainsonine plus paclitaxel. (E) The cells were treated with paclitaxel $(0,3,6$ and $10 \mathrm{ng} / \mathrm{ml})$ for $24 \mathrm{~h}$ and the expression of $\mathrm{NF}-\kappa \mathrm{B} / \mathrm{p} 65$ in nuclear and cytoplasmic regions was determined by western blot analysis. (F) Nuclear and cytoplasmic extracts were prepared from cells treated with paclitaxel, swainsonine alone or in combination for $24 \mathrm{~h}$. Western blot analysis was used to determine the expression of $\mathrm{NF}-\kappa \mathrm{B} / \mathrm{p} 65$ in nuclear and cytoplasmic regions.

is associated with the progression, metastasis and clinical outcome of a number of cancer types. Thus, the inhibition of Golgi $\alpha$-mannosidase II has shown clinical potential in cancer treatment (21). Recently, numerous studies have suggested through the expression changes of apoptosis-related genes in vivo that swainsonine has potential for treating glioma and gastric carcinoma through the mechanism of induced apoptosis. Additionally, initial clinical research has confirmed a clear curative effect against pate malignant tumor and chest and abdominal lymphangioma (14-16). Evidence indicates that swainsonine is a potent anticancer agent and the anticancer action of swainsonine may result from the co-operation of a number of pathways. However, a phase II clinical trial of GD0039 (a hydrochloride salt of swainsonine) in 17 patients with renal carcinoma was discouraging (22). The reason for the disparity is unknown; however, it may be due to the different types of cancer. Despite the controversy surrounding its efficacy, swainsonine may be considered a satisfactory candidate drug for cancer targeting therapy. Further studies regarding this agent may possibly lead to its clinical application.

Although numerous literature exists regarding swainsonine acting on hepatoma cells, these studies applied swainsonine on hepatoma cells for the related research of oligosaccharides $(23,24)$. Reports investigating the roles of swainsonine in antiHCC therapy, particularly regarding the molecular mechanisms do not exist. Therefore in this study, we focused on investigating the direct anti-HCC action of swainsonine and the potential pathways involved. Furthermore, we investigated whether swainsonine chemosensitizes HCC to paclitaxel-induced cytotoxicity via attenuating the constitutive activation of $N F-\kappa B$.
Cancer is known as a disease for deregulating signal transduction pathways that regulate fundamental processes such as cell growth (25). In the last few years, accumulating data also suggest that the regulation of intracellular growth signaling events may be controlled by the progression of the cell cycle and apoptosis (26). In addition, current cytotoxic therapies such as chemotherapy, radiotherapy or immunotherapy critically depend on inducing cell cycle arrest and cancer cell apoptosis (27-29). Hence, a therapeutic strategy aimed at the cell cycle and apoptosis is expected to provide an effective treatment for cancer.

In this research, we demonstrated that the treatment of HCC cells with swainsonine reduces the viability of hepatoma cells, but induced little or no cytotoxicity to hepatocytes. Furthermore, using flow cell cytometric analysis techniques, we showed that swainsonine inhibited the growth of MHCC97-H cells accompanied by increasing the number of cells in the G0/G1 phases and decreasing the number of cells in the $\mathrm{S}$ and $\mathrm{G} 2 / \mathrm{M}$ phases. Cell cycle is regulated by the concerted actions of cyclins, Cdks and Cdk inhibitors (30). The physiological process of cell cycle from phase G1 to $\mathrm{S}$ is controlled at several steps by cyclin D1, cyclin E, Cdk2 and Cdk4 (31). As cell cycle suppressor molecules, the important function of p21 and p27 is to induce cell cycle arrest by forming heterotrimeric complexes with G1-S Cdks and cyclins to inhibit their activity (32). Our western blot results indicated that cyclin D1, cyclin E, Cdk2 and Cdk4 were downregulated and that p21 and p27 were upregulated by swainsonine treatment. However, our results are not in total agreement with data obtained by Sun et al (16), who reported that swainsonine induced tumor cell 
arrest in $\mathrm{S}$ phase. This indicates that swainsonine may induce different types of cell cycle arrest in different cell types. Cell cycle regulation is complicated and cell-specific. Further study is essential to disclose the mechanism of swainsonine on cell cycle arrest.

There is growing evidence that apoptosis failure may be involved in the pathogenesis of cancer. The ability of tumor cells to evade the engagement of apoptosis may also play a significant role in their resistance to conventional therapeutic regimens (33). Therefore, a therapeutic strategy aimed at specifically triggering apoptosis in cancer cells may have a potential therapeutic effect. In our study, we observed a significant induction of apoptosis in swainsonine-treated MHCC97-H cells, indicating that swainsonine potentiates the apoptotic machinery. There are two classic apoptotic pathways in mammalian cells, namely, the mitochondria-mediated apoptotic and the death receptor-mediated apoptotic pathway (34). Both apoptotic pathways are gene-regulated. We observed that the treatment of MHCC97-H cells with swainsonine resulted in the upregulation of Bax levels, along with the reduction in Bcl-2. However, the expression levels of Fas and Fas-L were not changed significantly. The results showed that swainsonine induced apoptosis in MHCC97-H cells mainly through the mitochondria-mediated pathway.

Constitutive activation of $\mathrm{NF}-\kappa \mathrm{B}$ has been described in a number of solid tumors, including HCC. This activation is involved in the regulation of transcription of various genes involved in cell growth and apoptosis (35). Depending on the specific role of $\mathrm{NF}-\kappa \mathrm{B}$ in the cell cycle and apoptosis, we investigated whether $\mathrm{NF}-\kappa \mathrm{B}$ was involved in the inhibition of HCC cell growth by swainsonine. Our results showed that swainsonine-induced inhibition of cell growth, cell cycle arrest and apoptosis were accompanied by a significant inactivation

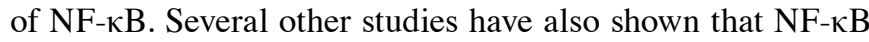
induces the expression of cyclin D1, Bcl-2 and Bcl-xL (36). Our results showed that the expression levels of cyclin D1 and Bcl-2 were reduced when cells were treated with swainsonine and this occurred at least partially via the inactivation of $\mathrm{NF}-\kappa \mathrm{B}$ signaling. Therefore, it may be suggested that the inhibition of HCC cell growth by swainsonine is possibly mediated through the inhibition of NF- $\kappa$ B.

In addition, the activation of $\mathrm{NF}-\kappa \mathrm{B}$ plays an important role in the development of drug resistance. Currently, many researchers have focused their efforts on exploring chemosensitizing drugs, with $\mathrm{NF}-\kappa \mathrm{B}$ suppression activity, used in combination with other chemotherapeutic agents for effective management of HCC (37). Paclitaxel is a useful chemotherapeutic drug for the treatment of patients with a variety of malignant tumors (38). However, chemoresistance due to paclitaxel-induced NF- $\mathrm{KB}$ activation is an important cause of the limits of paclitaxel efficacy (39). Several reports have shown the advantage of combination treatments (40). In this study, we showed the chemosensitizing effect of swainsonine on MHCC97-H cells to paclitaxel toxicity in vitro and in vivo. Combination treatment was significantly superior to paclitaxel or swainsonine alone. In this study, we also revealed that combination treatment of paclitaxel and swainsonine is associated with the inhibition of $\mathrm{NF}-\kappa \mathrm{B}$ activity.

In conclusion, the present study demonstrated that swainsonine significantly inhibits the growth of HCC cells by causing cell cycle arrest and the induction of apoptosis. Swainsonine causes G1 phase cell cycle arrest and the induction of apoptosis by altering the cell cycle and expression of associated survival proteins. Furthermore, our study suggests a role of swainsonine in chemosensitizing $\mathrm{HCC}$ cells to the cytotoxic effects of paclitaxel. Suppression of $\mathrm{NF}-\kappa \mathrm{B}$ activation may be one of the mechanisms involved in the swainsonine-induced inhibition of growth and chemosensitizing effects in HCC cells. Our study provides important data with which to evaluate the possible clinical application of swainsonine as a novel anti-HCC agent. Further studies are in progress in our laboratory to provide additional data for clinical application.

\section{Acknowledgements}

The authors would like to thank Juan Li and Jintao Hu for their excellent technical assistance. The Chinese National Natural Science Foundation grant no. 81170419 funded this study.

\section{References}

1. Lin H, van den Esschert J, Liu C and van Gulik TM: Systematic review of hepatocellular adenoma in China and other regions. J Gastroenterol Hepatol 26: 28-35, 2011.

2. Villanueva A and Llovet JM: Targeted therapies for hepatocellular carcinoma. Gastroenterology 140: 1410-1426, 2011.

3. Yang JD and Roberts LR: Hepatocellular carcinoma: a global view. Nat Rev Gastroenterol Hepatol 7: 448-458, 2010.

4. Sanoff HK, Bernard S, Goldberg RM, et al: Phase II study of capecitabine, oxaliplatin, and cetuximab for advanced hepatocellular carcinoma. Gastrointest Cancer Res 4: 8-83, 2011.

5. Okano J, Nagahara T, Matsumoto K and Murawaki Y: The growth inhibition of liver cancer cells by paclitaxel and the involvement of extracellular signal-regulated kinase and apoptosis. Oncol Rep 17: 1195-1200, 2007.

6. Caicedo-Granados EE, Wuertz BR, Marker PH, Lee GS and Ondrey FG: The effect of indomethacin on paclitaxel sensitivity and apoptosis in oral squamous carcinoma cells: the role of nuclear factor- $\mathrm{\kappa B}$ inhibition. Arch Otolaryngol Head Neck Surg 137: 799-805, 2011.

7. Sreekanth CN, Bava SV, Sreekumar E and Anto RJ: Molecular evidences for the chemosensitizing efficacy of liposomal curcumin in paclitaxel chemotherapy in mouse models of cervical cancer. Oncogene 30: 3139-3152, 2011.

8. Kelly MG, Alvero AB, Chen R, et al: TLR-4 signaling promotes tumor growth and paclitaxel chemoresistance in ovarian cancer. Cancer Res 66: 3859-3868, 2006.

9. Baines AT, Xu D and Der CJ: Inhibition of Ras for cancer treatment: the search continues. Future Med Chem 3: 1787-1808, 2011.

10. Stronach EA, Chen M, Maginn EN, Agarwal R, Mills GB, Wasan $\mathrm{H}$ and Gabra H: DNA-PK mediates AKT activation and apoptosis inhibition in clinically acquired platinum resistance. Neoplasia 13: 1069-1080, 2011.

11. Luedde T and Schwabe RF: NF- $\kappa B$ in the liver - linking injury, fibrosis and hepatocellular carcinoma. Nat Rev Gastroenterol Hepatol 8: 108-118, 2011.

12. Luqman S and Pezzuto JM: NFkappaB: a promising target for natural products in cancer chemoprevention. Phytother Res 24: 949-963, 2010.

13. Nogueira L, Ruiz-Ontañon P, Vazquez-Barquero A, Moris F and Fernandez-Luna JL: The NFאB pathway: a therapeutic target in glioblastoma. Oncotarget 2: 646-653, 2011.

14. Goss PE, Baptiste J, Fernandes B, Baker M and Dennis JW: A phase I study of swainsonine in patients with advanced malignancies. Cancer Res 54: 1450-1457, 1994.

15. Santos FM, Latorre AO, Hueza IM, Sanches DS, Lippi LL, Gardner DR and Spinosa HS: Increased antitumor efficacy by the combined administration of swainsonine and cisplatin in vivo. Phytomedicine 18: 1096-1101, 2011.

16. Sun JY, Yang H, Miao S, et al: Suppressive effects of swainsonine on C6 glioma cell in vitro and in vivo. Phytomedicine 16: 1070-1074, 2009. 
17. You N, Liu W, Zhong X, et al: Tg737 inhibition results in malignant transformation in fetal liver stem/progenitor cells by promoting cell-cycle progression and differentiation arrest. Mol Carcinog 51: 659-673, 2012.

18. Alves RC, Alves D, Guz B, et al: Advanced hepatocellular carcinoma. Review of targeted molecular drugs. Ann Hepatol 10: 21-27, 2011

19. Karikas GA: Anticancer and chemopreventing natural products: some biochemical and therapeutic aspects. J BUON 15: 627-638, 2010.

20. Tulsiani DR and Touster O: Swainsonine, a potent mannosidase inhibitor, elevates rat liver and brain lysosomal alpha-D-mannosidase, decreases Golgi alpha-D-mannosidase II, and increases the plasma levels of several acid hydrolases. Arch Biochem Biophys 224: 594-600, 1983.

21. van den Elsen JM, Kuntz DA and Rose DR: Structure of Golg alpha-mannosidase II: a target for inhibition of growth and metastasis of cancer cells. EMBO J 20: 3008-3017, 2001.

22. Shaheen PE, Stadler W, Elson P, Knox J, Winquist E and Bukowski RM: Phase II study of the efficacy and safety of oral GD0039 in patients with locally advanced or metastatic renal cell carcinoma. Invest New Drugs 23: 577-581, 2005.

23. Yanagida K, Natsuka S and Hase S: Structural diversity of cytosolic free oligosaccharides in the human hepatoma cell line, HepG2. Glycobiology 16: 294-304, 2006.

24. Yeo TK, Yeo KT, Parent JB and Olden K: Swainsonine treatment accelerates intracellular transport and secretion of glycoproteins in human hepatoma cells. J Biol Chem 260: 2565-2569, 1985.

25. Williams GH and Stoeber K: The cell cycle and cancer. J Pathol 226: 352-364, 2012.

26. Fang J, Yu Z, Lian M, Ma H, Tai J, Zhang L and Han D: Knockdown of zinc finger protein, X-linked (ZFX) inhibits cell proliferation and induces apoptosis in human laryngeal squamous cell carcinoma. Mol Cell Biochem 360: 301-307, 2012.

27. Hu W, Shen T and Wang MH: Cell cycle arrest and apoptosis induced by methyl 3,5-dicaffeoyl quinate in human colon cancer cells: Involvement of the PI3K/Akt and MAP kinase pathways. Chem Biol Interact 194: 48-57, 2011.

28. Palumbo C, Bei R, Procopio A and Modesti A: Molecular targets and targeted therapies for malignant mesothelioma. Curr Med Chem 15: 855-867, 2008.

29. Rahbari NN, Mehrabi A, Mollberg NM, Müller SA, Koch M, Büchler MW and Weitz J: Hepatocellular carcinoma: current management and perspectives for the future. Ann Surg 253: 453-469, 2011.
30. Johansson M and Persson JL: Cancer therapy: targeting cell cycle regulators. Anticancer Agents Med Chem 8: 723-731, 2008.

31. Chang HR, Lian JD, Lo CW, Chang YC, Yang MY and Wang CJ: Induction of urothelial proliferation in rats by aristolochic acid through cell cycle progression via activation of cyclin D1/cdk4 and cyclin E/cdk2. Food Chem Toxicol 44: 28-35, 2006.

32. Chen K, Perez-Stable C, D'Ippolito G, Schiller PC, Roos BA and Howard GA: Human bone marrow-derived stem cell proliferation is inhibited by hepatocyte growth factor via increasing the cell cycle inhibitors p53, p21 and p27. Bone 49: 1194-1204, 2011.

33. Sayers TJ: Targeting the extrinsic apoptosis signaling pathway for cancer therapy. Cancer Immunol Immunother 60: 1173-1180, 2011.

34. Chowdhury I, Tharakan B and Bhat GK: Current concepts in apoptosis: the physiological suicide program revisited. Cell Mol Biol Lett 11: 506-525, 2006.

35. He G and Karin M: NF- $\kappa$ B and STAT3 - key players in liver inflammation and cancer. Cell Res 21: 159-168, 2011.

36. Deeb D, Gao X, Dulchavsky SA and Gautam SC: CDDO-Me inhibits proliferation, induces apoptosis, down-regulates Akt, mTOR, NF-kappaB and NF-kappaB-regulated antiapoptotic and proangiogenic proteins in TRAMP prostate cancer cells. J Exp Ther Oncol 7: 31-39, 2008.

37. Ma Y, Wang J, Liu L, et al: Genistein potentiates the effect of arsenic trioxide against human hepatocellular carcinoma: role of Akt and nuclear factor- $\kappa$ B. Cancer Lett 301: 75-84, 2011.

38. Matsubara J, Shimada Y, Kato K, et al: Phase II study of bolus 5-fluorouracil and leucovorin combined with weekly paclitaxel as first-line therapy for advanced gastric cancer. Oncology 81: 291-297, 2011.

39. Fujiwara Y, Furukawa K, Shimada Y, et al: Combination paclitaxel and inhibitor of nuclear factor $\kappa \mathrm{B}$ activation improves therapeutic outcome for model mice with peritoneal dissemination of pancreatic cancer. Pancreas 40: 600-607, 2011.

40. Pasqualetti G, Ricciardi S, Mey V, Del Tacca M and Danesi R: Synergistic cytotoxicity, inhibition of signal transduction pathways and pharmacogenetics of sorafenib and gemcitabine in human NSCLC cell lines. Lung Cancer 74: 197-205, 2011. 